\title{
SCIENTIFIC REPORTS

\section{OPEN The interplay between chondrocyte spheroids and mesenchymal stem cells boosts cartilage regeneration within a 3D natural-based hydrogel}

Received: 3 April 2019
Accepted: 25 September 2019 Published online: 10 October 2019

\author{
Annachiara Scalzone ${ }^{1}$, Ana M. Ferreiraa ${ }^{1}$, Chiara Tonda-Turo ${ }^{2}$, Gianluca Ciardelli $i^{2}$, \\ Kenny Dalgarno ${ }^{1} \&$ Piergiorgio Gentile $\mathbb{B}^{1}$
}

Articular cartilage (AC) lacks the ability to self-repair and cell-based approaches, primarily based on using chondrocytes and mesenchymal stem cells (MSCs), are emerging as effective technology to restore cartilage functionality, because cells synergic functionality may support the maintenance of chondrogenic phenotype and promote extracellular matrix regeneration. This work aims to develop a more physiologically representative co-culture system to investigate the influence of MSCs on the activity of chondrocytes. A thermo-sensitive chitosan-based hydrogel, ionically crosslinked with $\beta$ glycerophosphate, is optimised to obtain sol/gel transition at physiological conditions within 5 minutes, high porosity with pores diameter $<30 \mu \mathrm{m}$, and in vitro mechanical integrity with compressive and equilibrium Young's moduli of $37 \mathrm{kPa}$ and $17 \mathrm{kPa}$, respectively. Live/dead staining showed that after 1 and 3 days in culture, the encapsulated MSCs into the hydrogels are viable and characterised by roundlike morphology. Furthermore chondrocyte spheroids, seeded on top of gels that contained either MSCs or no cells, show that the encapsulated MSCs stimulate chondrocyte activity within a gel co-culture, both in terms of maintaining the coherence of chondrocyte spheroids, leading to a larger quantity of CD44 (by immunofluorescence) and a higher production of collagen and glycosaminoglycans (by histology) compared with the mono-culture.

Articular cartilage (AC) is responsible for providing a load bearing and low friction interface between joint surfaces and is characterised by lack of ability to self-repair ${ }^{1}$. In order to stimulate repair, tissue engineering (TE) strategies combine cells and biomaterials to promote cartilage regeneration in vitro, with the cell type and culture environment important in modulating the degree of achievable regeneration of new tissue ${ }^{2}$. The most explored cell sources in this field are autologous chondrocytes and mesenchymal stem cells (MSCs) ${ }^{3}$. While chondrocytes are widely used in cell-based cartilage regenerative approaches, the limited availability of donor sites and low yield of isolated cells from autologous tissue (1-5\% of the total tissue volume), remain an issue ${ }^{4,5}$. Oppositely, MSCs are non-haematopoietic and multipotent adult stem cells that can be easily harvested from a number of human tissues ${ }^{6}$. In recent years, MSCs and chondrocytes co-cultures have grown in interest as offering a promising approach in cartilage TE, providing the intercellular signals considered important in regulating cell behaviour $^{7}$. When in co-culture, MSCs secrete trophic or cell-cell communication factors to promote proliferation and to delay de-differentiation of chondrocytes, while chondrocytes can induce chondrogenic differentiation of MSCs in the absence of other stimuli ${ }^{8}$. Co-culture systems are complex and have various mechanisms of action, as direct (cell to cell contact) and indirect (with cell types physically separated) in vitro co-culture offering different intercellular interactions. While indirect contact in vitro co-cultures have been used to evaluate the effect of cytokines secreted by one cell type on the other via autocrine or paracrine signalling; direct physical contact in vitro facilitates cell-cell interactions though surface receptors, enhancing the transduction of the molecular signals coordinating the chondrogenic differentiation (ie. VEGF-164 $\alpha$, TIMP-1, -2 and MMP-13) ${ }^{9-11}$.

\footnotetext{
${ }^{1}$ School of Engineering, Newcastle University, Claremont Road, Newcastle upon Tyne, NE1 7RU, United Kingdom. ${ }^{2}$ Department of Mechanical and Aerospace Engineering (DIMEAS), Politecnico di Torino Corso Duca degli Abruzzi 29, Turin, 10129, Italy. Correspondence and requests for materials should be addressed to P.G. (email: piergiorgio. gentile@ncl.ac.uk)
} 

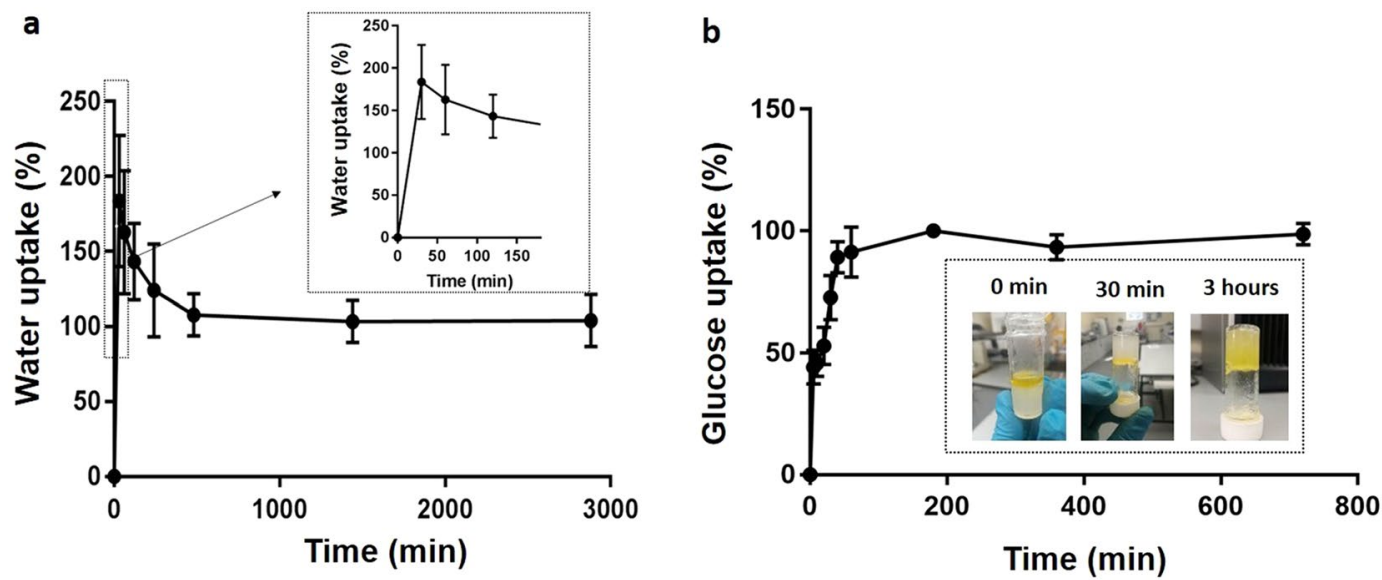

Figure 1. (a) Water uptake study of $\mathrm{CH} / \mathrm{BGP}$ at different time points. (b) $\mathrm{CH} / \mathrm{BGP}$ glucose uptake up to $720 \mathrm{~min}$. Insert: qualitative analysis of nutrient diffusion throughout the hydrogel.

The aim of the work presented in this paper was to develop a more physiologically representative co-culture system to investigate the influence of MSCs on the activity of chondrocytes. In order to do this we have encapsulated cells in a natural hydrogel, able to mimic the extracellular milieu which contains physical and chemical cues for cell-driven in vitro hyaline-type cartilage tissue development ${ }^{12}$. Smart stimuli-sensitive polymeric hydrogels, including temperature-sensitive and $\mathrm{pH}$ sensitive ones, have been widely exploited in regenerative medicine as these do not need any toxic chemical reagents to trigger sol-gel transition ${ }^{13}$. In this work, a thermo-sensitive chitosan $(\mathrm{CH})$ and $\beta$-glycerophosphate (BGP) hydrogel, previously explored in different biomedical applications (e.g. local drug delivery and nerve, bone and cartilage tissue engineering) was optimised as an in vitro 3D system for MSCs and chondrocytes co-culture ${ }^{14-16}$. Chitosan's structure and characteristics are similar to those of glycosaminoglycans (GAGs) present in the native cartilage, but it gels slowly ${ }^{17}$. However, the combination of chitosan with BGP, a chemical compound used in the body to transport minerals, allows fast chemical crosslinking such that within 5 minutes a thermally and mechanically stable hydrogel is obtained. The goal of this work has been to use the gel to encapsulate MSCs to prove its cytocompatibility and, then, to seed a chondrocyte spheroid on top of the gel, in order to evaluate if the presence of MSCs could influence the behaviour of the chondrocytes over an extended culture period.

\section{Results}

Gelation time and thermal irreversibility. Tube inverted method evidenced that CH/BGP system resulted to be liquid at room temperature up to $2 \mathrm{~h} 45 \mathrm{~min} \pm 10 \mathrm{~min}$, while it became a gel within $5 \pm 1 \mathrm{~min}$ and $2 \pm 0.5 \mathrm{~min}$ at $37^{\circ} \mathrm{C}$ and $50^{\circ} \mathrm{C}$ respectively. The hydrogel thermo-irreversibility was demonstrated maintaining the gel state after keeping the $\mathrm{CH} / \mathrm{BGP}$ system at $4^{\circ} \mathrm{C}$ where it maintained the structural stability without becoming liquid (Fig. S2).

Water uptake and nutrients diffusion. The water uptake ability of the chitosan-based hydrogel at $37^{\circ} \mathrm{C}$ is shown in Fig. 1a. The samples displayed an initial rapid water uptake of $184 \pm 44 \%$ within 30 min. Then, the water uptake over time achieving a value of about $108 \pm 14 \%$ within $8 \mathrm{~h}$. After stabilisation, a water uptake plateau was observed until $48 \mathrm{~h}$ after the immersion of the samples in Dulbecco's phosphate-buffered saline (DPBS) $(101 \pm 20 \%)$. Moreover, Fig. 1b shows the nutrients diffusion through the hydrogel via absorption of glucose dye solution. Particularly, after $30 \mathrm{~min}$ a superficial uptake of the dye from the gel appeared, while after $3 \mathrm{~h}$ the gel presented a homogenous yellow colour due to the complete dye uptake. The hydrogel ability to release nutrients over time was evaluated in fluorescence up to $48 \mathrm{~h}$ : a rapid 2-(N-(7-Bitrobenz-2-oxa-1,3-diazol-4-yl) Amino)2-Deoxyglucose (2-NBDG) uptake was found within the first $3 \mathrm{~h}$, reaching a close-to-maximum uptake plateau at $190 \pm 10 \mathrm{~min}$.

Chemical characterisation. Fourier Transformed Infrared analysis in Attenuated Total Reflectance (FTIR-ATR) spectra were measured for individual components (BGP and CH) as well as CH/BGP hydrogels before and after water uptake to analyse the chemical compositions (Fig. 2a). BGP spectrum evidenced a small peak at $1078 \mathrm{~cm}^{-1}$, characteristic of C-O bond, and a sharpen peak at $963 \mathrm{~cm}^{-1}$, that was characteristic of P-O bonds. The fundamental peaks of $\mathrm{CH}$ spectrum were $\mathrm{O}-\mathrm{H}$ and $\mathrm{N}-\mathrm{H}$ overlapping band, observed in the range of $3600-3100 \mathrm{~cm}^{-1}$, the stretching vibration of aliphatic groups $\left(-\mathrm{CH}_{2}\right.$ and $\left.-\mathrm{CH}_{3}\right)$, observed at $2970-2880 \mathrm{~cm}^{-1}$, primary and secondary amide bands $(\mathrm{C}=\mathrm{O}$ and $\mathrm{N}-\mathrm{H})$ appearing at 1656 and $1601 \mathrm{~cm}^{-1}$, peaks associated with $\mathrm{OH}$ oscillations and C-O stretching in the range of 1500 and $1200 \mathrm{~cm}^{-1}$ and, finally, the bridge (-O-) stretch of the glucosamine residues in the range $1200-800 \mathrm{~cm}^{-1}$ and a broad band at $660 \mathrm{~cm}^{-1}$ which was related with $\mathrm{O}=\mathrm{C}-\mathrm{N}$ vibrations. Compared to $\mathrm{CH}$, the $\mathrm{CH} / \mathrm{BGP}$ spectrum exhibited shift of the primary and secondary amide band respectively from 1656 to $1663 \mathrm{~cm}^{-1}$ and from 1601 to $1567 \mathrm{~cm}^{-1}$ (Fig. 2a left insert) and a less intensity of $\mathrm{C}=\mathrm{O}$, $\mathrm{O}-\mathrm{H}$ and $\mathrm{N}-\mathrm{H}$ stretching peaks. Furthermore, two new chemical bands appeared at $1000 \mathrm{~cm}^{-1}$ and $800 \mathrm{~cm}^{-1}$ indicating aliphatic $\mathrm{P}-\mathrm{O}-\mathrm{C}$ stretching while the new band at $1050 \mathrm{~cm}^{-1}$ was characteristic of the $-\mathrm{PO}_{4}{ }^{2-}$ and a 


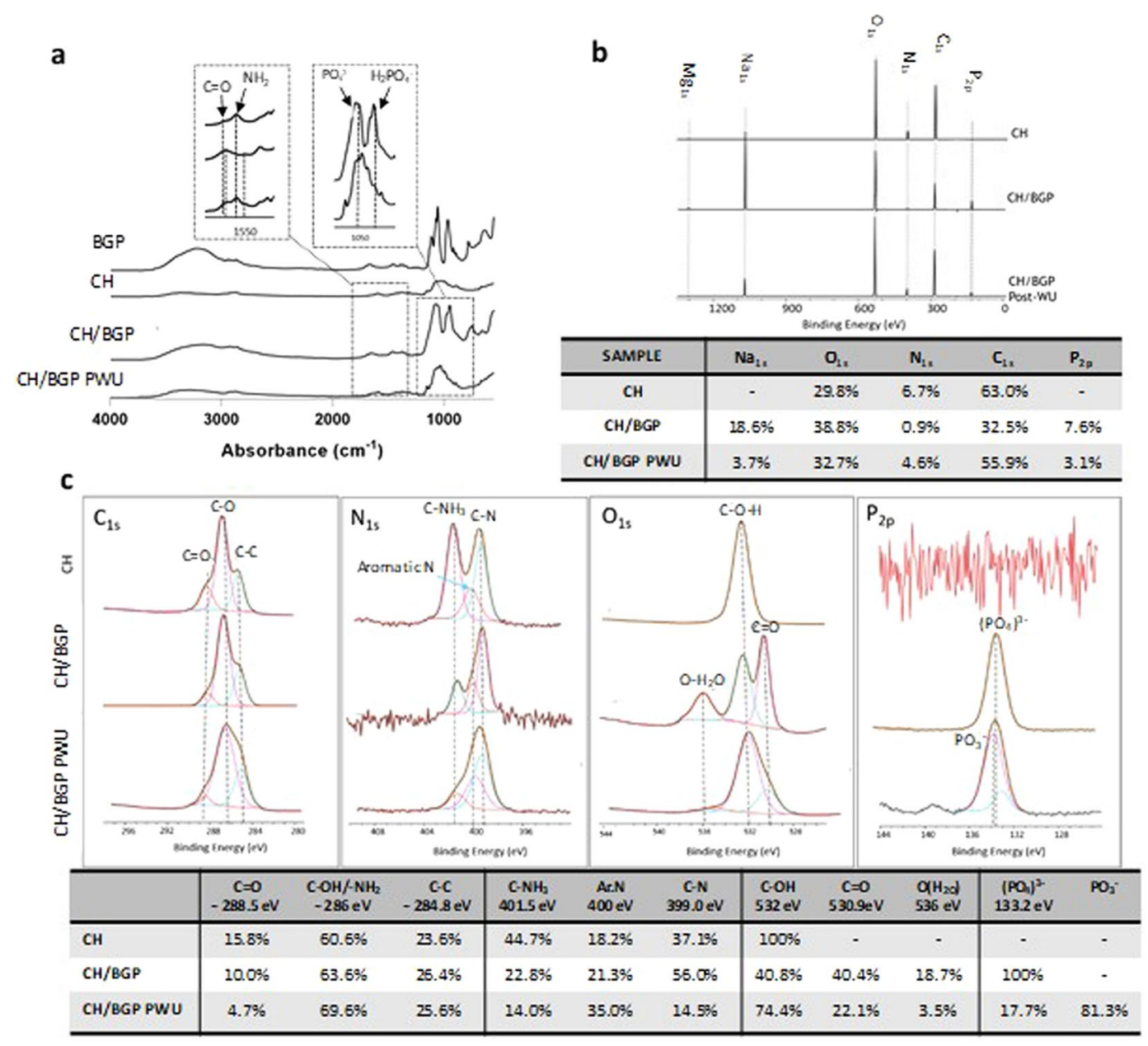

Figure 2. (a) FTIR-ATR spectra of BGP, CH, CH/BGP samples before and after water uptake in the range $4000-550 \mathrm{~cm}^{-1}$. (b) XPS spectra survey of BGP, CH, CH/BGP samples before and after water uptake and the relative atomic percentage of the main elements $\left(\mathrm{Na}_{1 s}, \mathrm{O}_{1 s}, \mathrm{~N}_{1}, \mathrm{C}_{1 \mathrm{~s}}, \mathrm{P}_{2 \mathrm{p}}\right)$. (c) XPS high resolution spectra, showing the deconvolution peaks deconvolution for $\mathrm{CH}$ and $\mathrm{CH} / \mathrm{BGP}$ samples before and after water uptake (Post-WU).

new shoulder at $920 \mathrm{~cm}^{-1}$ characteristic of the $\mathrm{H}_{2} \mathrm{PO}_{4}{ }^{-}$(Fig. 2a right insert) ${ }^{18}$. After water uptake tests CH/BGP spectrum showed more similarities with the $\mathrm{CH}$ spectrum, where BGP characteristic peaks decreased in intensity. Figure 2b shows X-ray Photoelectron Spectroscopy (XPS) analysis reporting the survey spectra and relative atomic percentage of the main elements. The results showed the presence of $\mathrm{O}_{1 s}$, nitrogen $\mathrm{N}_{1 \mathrm{~s}}$ and carbon $\mathrm{C}_{1 \mathrm{~s}}$ that were main characteristics of chitosan, in all the samples. The hydrogel formation led an evident decrease of $\mathrm{N}_{1 \mathrm{~s}}$ and $\mathrm{C}_{1 \mathrm{~s}}$ content from $6.7 \%$ to $0.9 \%$, and from $63.0 \%$ to $32.5 \%$ respectively (CH vs $\mathrm{CH} / \mathrm{BGP}$ ). Furthermore $\mathrm{Na}_{1 \text { s }}$ and $\mathrm{P}_{2 \mathrm{p}}$ peaks, $\mathrm{BGP}$ chemical elements, appeared after the hydrogel formation before $(18.6 \%$ and $7.6 \%)$ and after water uptake (3.7\% and 3.1\%). The re-increase of those percentage (from $0.9 \%$ to $4.6 \%$ in $\mathrm{N}_{1 \mathrm{~s}}$ and from $32.5 \%$ to $55.9 \%$ in $\mathrm{C}_{1 \mathrm{~s}}$ ) was evident after water uptake. High resolution spectra (Fig. 2c) evidenced changes in bonds percentage, such as: $\mathrm{C}=\mathrm{O}(288.5 \mathrm{eV})$ content decreased from $15.8 \%$ in $\mathrm{CH}$ to $10.0 \%$ and $4.7 \%$ respectively in $\mathrm{CH} / \mathrm{BGP}$ before and after water uptake; while $\mathrm{C}-\mathrm{OH}(532 \mathrm{eV})$ content decreased from $100 \%$ in the $\mathrm{CH}$ to $40.8 \%$ in $\mathrm{CH} / \mathrm{BGP}$ and re-increased up to $74.4 \%$ after water uptake. New bonds appeared after the hydrogel formation: $\mathrm{C}=\mathrm{O}(530.9 \mathrm{eV})$ with a content of $40.4 \%$ in $\mathrm{CH} / \mathrm{BGP}$ and $22.1 \%$ after water uptake; $\mathrm{O}\left(\mathrm{H}_{2} \mathrm{O}\right)(536 \mathrm{eV})$ with a $18.7 \%$ in $\mathrm{CH} / \mathrm{BGP}$ and a decrease to $3.5 \%$ after water uptake; $\mathrm{PO}_{4}{ }^{3-}(133.2 \mathrm{eV})$ decreased up to $17.7 \%$ after water uptake. Finally, $\mathrm{PO}^{3-}(134 \mathrm{eV})$ was present in the $\mathrm{CH} / \mathrm{BGP}$ samples after water uptake only.

CH/BGP hydrogel morphology. Environmental scanning electron microscope (E-SEM) analyses were performed to evaluate the influence of BGP addition on the morphology of the freeze-dried samples (Fig. 3a). Both samples (before and after water uptake) showed a porous structure with "open-cell" structure, high degree of interconnectivity and irregular pores shape. However, after water uptake, samples evidenced smoother pore walls than as-prepared samples, more evident at the highest magnification (1200x). Furthermore, the water uptake influenced the pore size of the samples (Fig. 3b): pores with a diameter below $10 \mu \mathrm{m}$ (64\% of pores) with an average pore size measured of $5.6 \pm 2.6 \mu \mathrm{m}$ in the as-prepared samples, while after water uptake most of the pores were in the range $5-20 \mu \mathrm{m}$ ( $86 \%$ of pores) with an average pore size of $11.5 \pm 4.1 \mu \mathrm{m}$. 


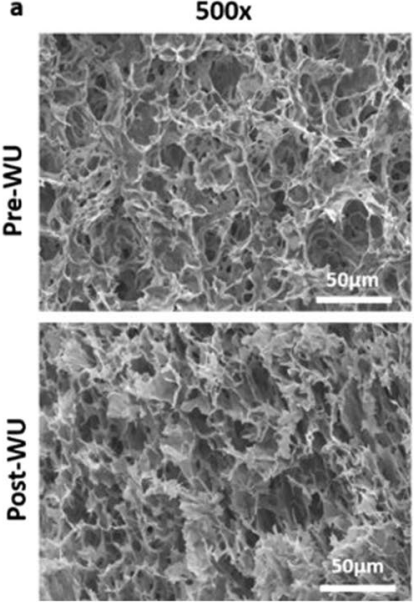

$1200 x$

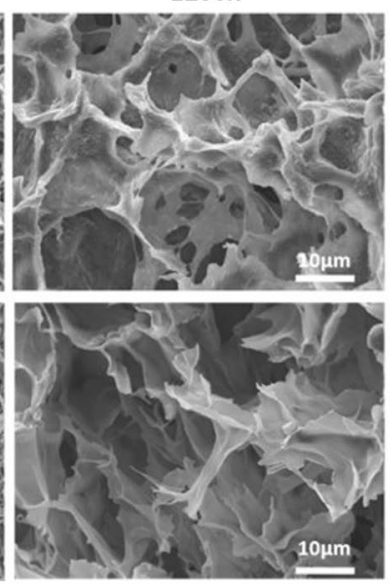

b

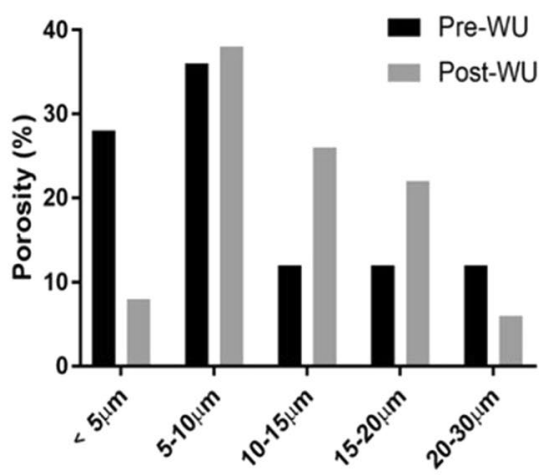

Figure 3. (a) ESEM images, representing cross-section microstructure of hydrogels before (Pre-WU) and post water-uptake (Post-WU) at 500x and 2000x magnification. (b) Distribution analysis of the pores within the ranges $<5 \mu \mathrm{m}, 5-10 \mu \mathrm{m}, 10-15 \mu \mathrm{m}, 15-20 \mu \mathrm{m}$ and $20-30 \mu \mathrm{m}$.

Mechanical properties. Under static compression it was calculated a compressive elastic Young's modulus of approximately $36 \pm 4.0 \mathrm{kPa}$, while for the dynamic stress relaxation test an Equilibrium Young's modulus of $17.4 \pm 0.8 \mathrm{kPa}$ was obtained after $1000 \mathrm{~s}$. Furthermore, from the computational analysis done by using Matlab software of the stress-relaxation curves, the following relaxation times $\tau_{1}=9.2 \pm 0.5 \mathrm{~s}, \tau_{2}=65.0 \pm 4.8 \mathrm{~s}$ and $\tau_{3}=450.0 \pm 35.7 \mathrm{~s}$ were calculated.

A further analysis on the mechanical properties has been performed by rheology. The linear viscoelastic region where storage modulus $\left(\mathrm{G}^{\prime}\right)$ and the Loss modulus $\left(\mathrm{G}^{\prime \prime}\right)$ were independent from the shear strain was measured through strain sweep test, determining a linear behaviour for 0.1 to $10 \%$ strain. Then, the amplitude of strain for following rheological measurements was set at $1 \%$. In the temperature sweep test, $G^{\prime}$ and $G^{\prime \prime}$ slightly decreased with the increase in temperature until the crossing point where $\mathrm{G}^{\prime}>\mathrm{G}^{\prime \prime}\left(\mathrm{T}=32 \pm 1^{\circ} \mathrm{C}\right)$ and the sol/gel transition occurred. With the time sweep test, the time required for solution gelification at $37^{\circ} \mathrm{C}$ (crossing point where $\mathrm{G}^{\prime}$ became higher than $\mathrm{G}^{\prime \prime}$ ) was measured and the found gelation times was $t_{\mathrm{S} / \mathrm{G}}=144 \pm 12 \mathrm{~s}$. Figure $\mathrm{S} 3$ shows the graphs obtained from the mechanical analysis.

Cytocompatibility evaluation. Live/dead staining showed that after 1 and 3 days in culture, the encapsulated MSCs into the hydrogels were viable and characterised by round-like morphology (Fig. 4a). Few dead (red) cells were observed. The immunostaining analysis (nuclei stained with 4', 6- diamidino-2-phenylindole (DAPI) and the cytoskeleton with rhodamine-phalloidin) confirmed that the thermo-sensitive hydrogels were compatible with a tendency of the MSCs to form agglomerates after 3 days of culture. Cells metabolic activity resulted to be non-statistically different from day 1 to day 7 (1230 $\pm 150 \mathrm{RFU}$ to $800 \pm 105 \mathrm{RFU}$ ) (Fig. 4b), demonstrating that materials mixing and gelation process did not adversely affect the viability of the encapsulated cells. Finally, Transmission Electron Microscope (TEM) analysis evidenced that the structure of the cells were not altered after the cell culture in the gel (Fig. 4c): at the ultrastructural level, MSC showed large nucleus (N) with abundant chromatin, little marginally condensed heterochromatin with the presence of nucleoli; cell organelles (endoplasmic reticulum (ER) and mitochondria (M)) were not altered and a slight vacuolisation of the cytoplasm was observed. The arrows indicate the endocytosis process for the BGP salt internalisation with the formation of vacuoles.

Neocartilage formation by MSCs-hACHs co-culture. Co-culture immunofluorescence analysis was performed after 7 and 14 days (Fig. 5a), staining cells nuclei in blue, Collagen Type II (Col II) fibres in red and $\mathrm{CD} 44$ in green. At day 7, the images report the hydrogel area around the spheroid and the spheroid itself. In co-culture condition, MSCs cells were homogenously dispersed within the gel while the chondrocyte spheroid was characterised by a round-like compact shape. A similar morphology of chondrocytes spheroid was found in the mono-culture, without chondrocytes migration into the hydrogel. CD44 was present within the spheroid and the hydrogel in the co-culture, and spread within the hydrogel in the mono-culture. Finally, in the co-culture conditions it was observed a small amount of Col II after 7 days of culture, while it was totally absent in the mono-culture. The results obtained on day 7 were compared with day 14 . Particularly, in the co-culture, a higher production of Col II and a larger quantity of CD44 were evidenced compared to the mono-culture. For the co-culture $3 \mathrm{D}$ volumetric stack images up to 28 days were reported, showing clearly the Col II production increase over the time (Fig. S5). Histological analyses at 28days are shown in Fig. 5b.

After 4 weeks of co-culture of human Articular Chondrocytes (hACHs) spheroid on a MSCs-laden hydrogel in chondrocytes proliferation medium, the production of GAGs and collagen were clearly visible and homogenously distributed, compared to the hACHs mono-culture, where GAGs and collagen production was found to be low and fragmented. Furthermore comparing MSCs spheroid within the MSc-laden hydrogel in both chondrogenic and chondrocytes growth media, immunofluorescence analysis confirmed the increment of Col II, 

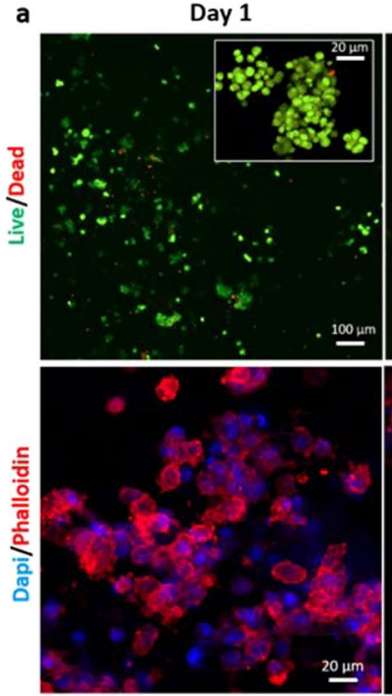

Day 3

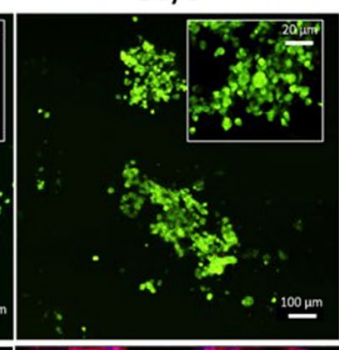

b

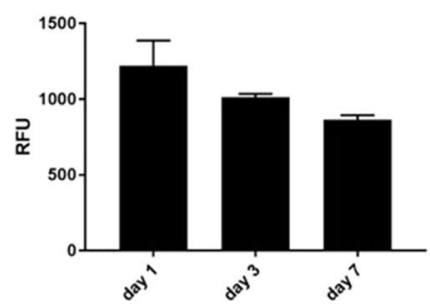

C

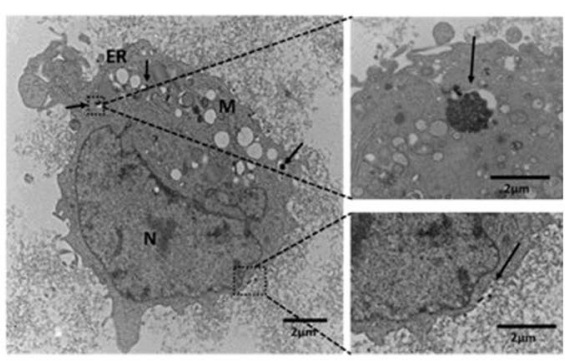

Figure 4. (a) Cytocompatibility evaluation of the MSCs-laden CH/BGP hydrogel: Live/Dead images of cells encapsulated in the CH/BGP hydrogel after $1 \mathrm{~d}$ and $3 \mathrm{~d}$ (green: live and red: dead cells) (top) and Immunostaining images of cells encapsulated in the CH/BGP hydrogel after $1 \mathrm{~d}$ and $3 \mathrm{~d}$ (blue: nuclei and red: cytoskeleton) (bottom). (b) PrestoBlue results for cells encapsulated in the hydrogel at $1 \mathrm{~d}, 3 \mathrm{~d}$ and $7 \mathrm{~d}$. RFU is referred to relative fluorescence units. No statistical differences were revealed. (c) TEM images of encapsulated MSCs in the hydrogel after $24 \mathrm{~h}$ incubation: black arrows indicates the salt uptake by cells, $\mathrm{N}$ labels nucleus, ER the endoplasmic reticulum and $\mathrm{M}$ mitochondria; in the inserts are shown magnifications of vacuole with salt (top) and endocytosis at cell surface process (bottom).

CD44 expression in the co-culture compared to mono-cultures (Fig. S6a) while histological analysis showed an enhancement of the collagen and GAGs accumulation when cells were in co-culture (Fig. S6b).

Co-culture morphological analysis. SEM investigation of the hydrogels surface and cross-sections revealed the presence of numerous cells entrapped within the matrix (Fig. 6). In co-culture condition chondrocytes cells, indicated by white arrows, were distributed within the pores of the matrix and exhibited a round-shaped morphology with a diameter $<20 \mu \mathrm{m}$. A similar structure with round-shaped cells was found for the hACHs mono-culture. Finally, a fiber-like matrix dispersed within the cells in both conditions was observed (bottom images).

\section{Discussion}

The CH/BGP formulation used in this work was optimised to obtain a gelation time of around 5 minutes at $37^{\circ} \mathrm{C}$ (Fig. S2), suitable for future in vivo applications with a neutral $\mathrm{pH}(7.0-7.2)$ able to avoid possibility of unwanted effect on cell survival or tissue irritation at the injection site. Previous works have reported that a salt concentration increase leads to a gelation time decrease ${ }^{19}$. Specifically, at low BGP concentration the electrostatic repulsions between positively charged chitosan chains do not allow coagulation of chitosan chains and a minimum amount of BGP salt is needed to neutralise the positive charge density on chitosan chains ${ }^{20}$. Over this threshold, electrostatic attractions between the phosphate groups of BGP molecules and the amine groups of chitosan allow extensive hydrogen bonding via $\mathrm{OH}-\mathrm{NH}$ and $\mathrm{O}-\mathrm{HN}$ in the chitosan chains ${ }^{15}$. Furthermore other parameters may affect the gelation time, such as the chitosan DD (95\% in this work), because the crosslink density increases between the phosphate groups and the amine groups ${ }^{21}$. A further key feature for injectable gels is the thermal stability of the formulation and the $\mathrm{CH} / \mathrm{BGP}$ hydrogel showed a thermo-irreversible behaviour at RT and at $4{ }^{\circ} \mathrm{C}$ without changes in its physical state. This property, as demonstrated by Ganji et al., is related to $\mathrm{BGP}$ amount and the relative crosslinking strength, as well as to the competition between the different molecular forces involved in gel formation: in fact hydrogen bonds are not temperature dependent and, thus, cooling the hydrogels does not affect the gel physical structure ${ }^{22,23}$. The hydrogel was hydrophilic, with a high capacity to hold water molecules and to assure a good nutrient transport. The decrease in the water uptake percentage shown in Fig. 1 was correlated to the over-saturation concentration of the salt, fundamental for the obtainment of a fast gelification, as demonstrated by FTIR-ATR, with a reduction in BGP characteristic peaks and by XPS with significant decrease in $\mathrm{Na}_{1 \mathrm{~s}}$ and $\mathrm{P}_{2 \mathrm{p}}$ intensity after WU tests ${ }^{24}$. SEM analysis showed that CH/BGP hydrogels possessed an open porous and interconnected network (more evident after WU due to the release of BGP excess). The hydrogels were characterised mainly by pores with diameters up to $30 \mu \mathrm{m}$, suitable for cell adhesion, nutrient absorption and diffusion (Fig. 3), and waste transport through the matrix ${ }^{25-27}$. In this work, the hydrogels showed a compressive Young's modulus of around $40 \mathrm{kPa}$ consistent with Kelly et al. that reported a compressive Young's modulus ranging from $100-1000 \mathrm{kPa}$ for native articular cartilage ${ }^{28}$. Other studies performed in literature found a compressive Young's modulus with values ranging from 5 to $20 \mathrm{kPa}^{29,30}$. Moreover, stress-relaxation behaviour of the prepared hydrogels was analysed, since it affects loads transfer and nutrients diffusion. The calculated 


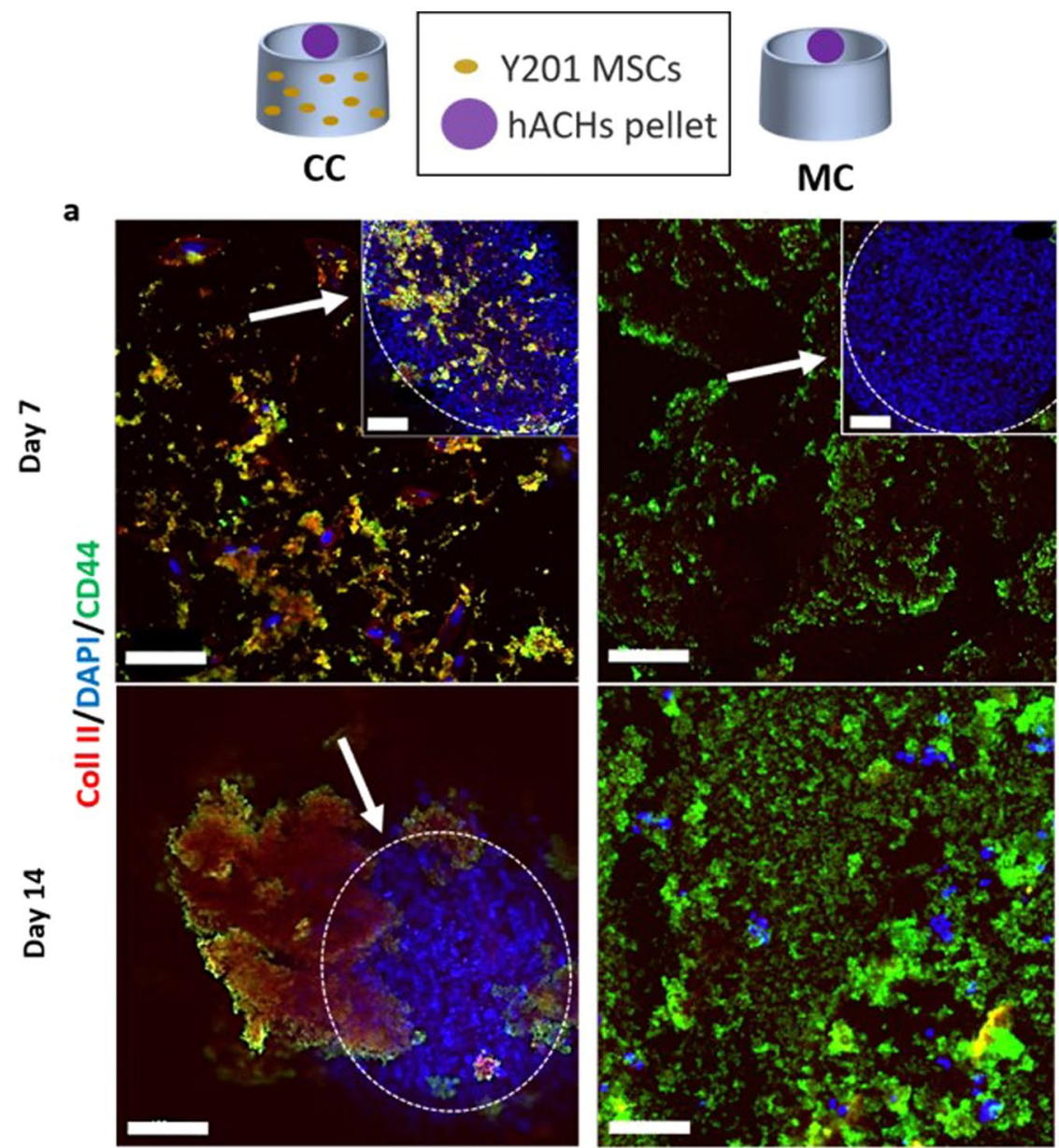

b
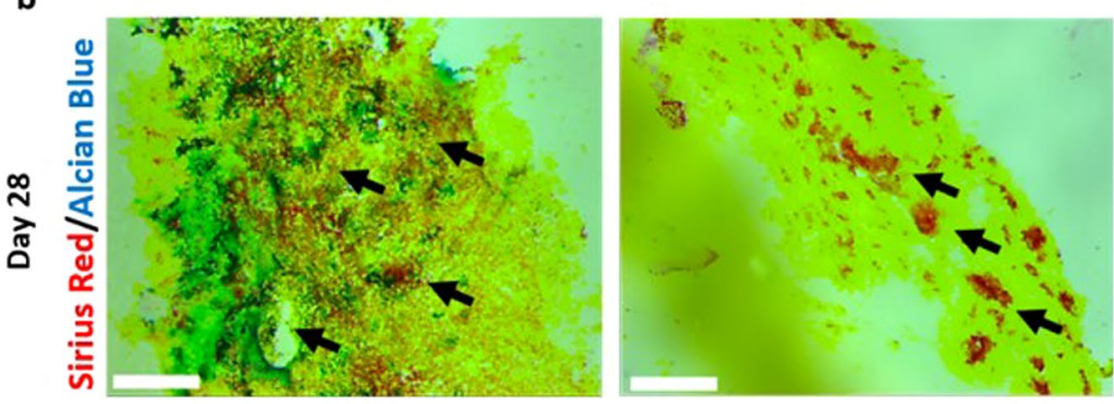

Figure 5. (a) Immunofluorescence staining at 7 (dashed line inserts represents spheroid area and white arrows are indicating at spheroids) and 14 days. Red staining for Col II, Blue for nuclei and Green for CD44 marker in co-culture (CC) (hACHs spheroid on MSCs-laden hydrogel) and mono-culture (MC) (hACHs spheroid on empty hydrogel). hACHs spheroids maintained their shape up to 14 days in the co-culture with MSCs while it disrupted after 7 days in the mono-culture due to the lack of MSCs stimuli. Bars $=100 \mu \mathrm{m}$. (b) Histological sections of the hydrogels after 28 culture days. GAGs were visualized by Alcian blue and Collagen by Sirius red staining respectively (Black arrows are indicating the area with higher production of collagen and GAGs). Bars $=200 \mu \mathrm{m}$.

relaxation times $\left(\tau_{1}=9.2 \pm 0.5 \mathrm{~s}, \tau_{2}=65.0 \pm 4.8 \mathrm{~s}\right.$ and $\left.\tau_{3}=450.0 \pm 35.7 \mathrm{~s}\right)$, calculated following the generalised Maxwell model, consisting in three relaxation times $\left(\tau_{1}=1-10 \mathrm{~s}, \tau_{2}=10-100 \mathrm{~s}\right.$ and $\left.\tau_{3}>1000 \mathrm{~s}\right)$ were in the range of those reported in literature for polymeric gels by Wagenseil et al. ${ }^{31}$. These relaxation times are related with the equilibrium modulus, defined as the stiffness of the gel as all the fluids flows out, that was found $\sim 17 \mathrm{kPa}$ and are in accordance with those reported for native cartilage, which varies with depth from the articular surface and other similar studies ${ }^{32,33}$.

In evaluating MSC viability TEM images revealed intact nuclei, mitochondria, and endoplasmic reticula, together with the presence of endocytosis at the cell surface indicating internalization of the salt into the cell in forms of vacuoles (Fig. 4). From the Live/Dead and the PrestoBlue analyses, the vacuolisation process did 


\section{Co-culture}
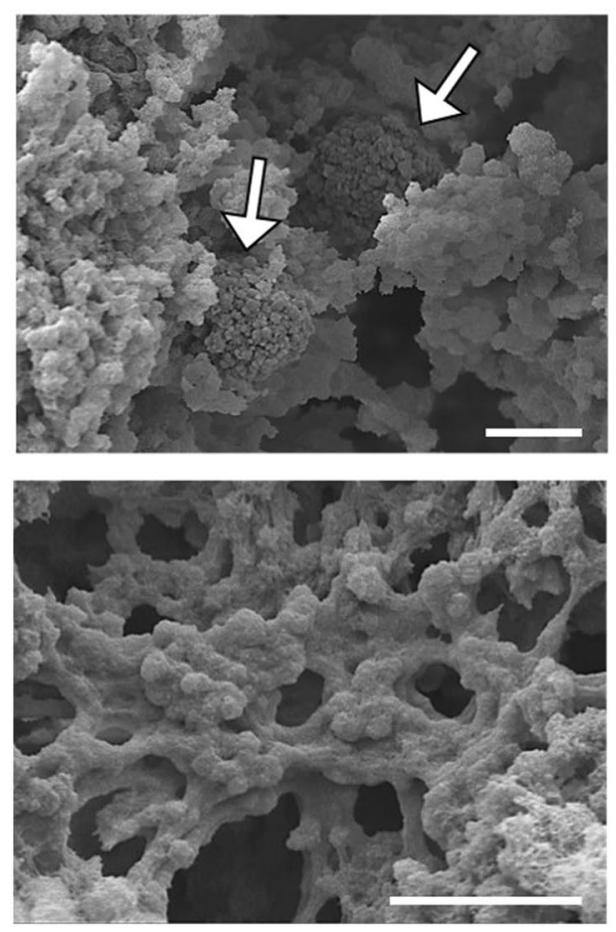

Mono-culture
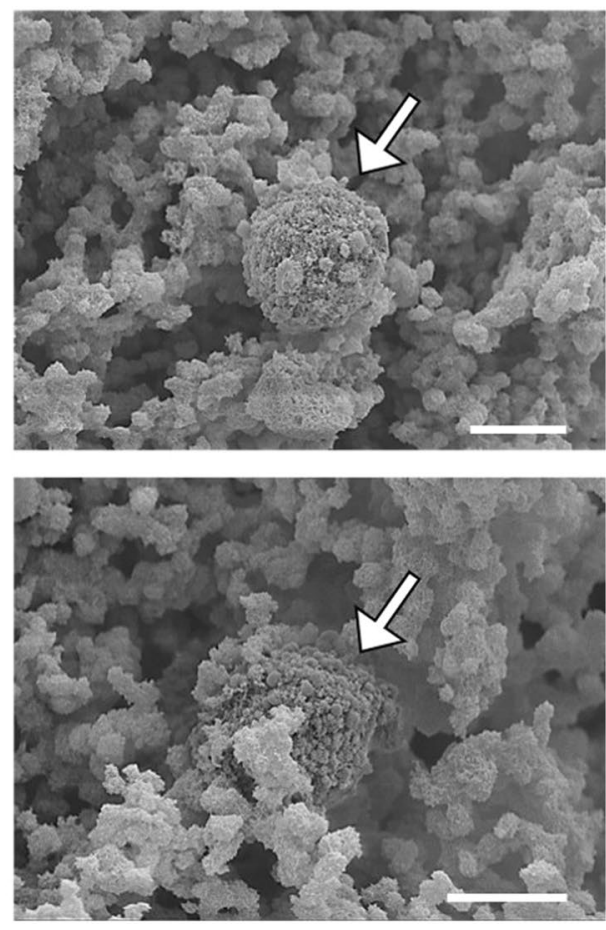

Figure 6. SEM micrographs of co-culture and mono-culture samples cross-sections at $45 \mathrm{~d}$ post-culture. Black arrows indicate chondrocyte cells. Scale bars: $10 \mu \mathrm{m}$.

not interfere negatively with cell viability and metabolic activity, potentially due to the consequent formation of a large autophagosome expelled through exocytosis ${ }^{34}$. These results are slightly in contrast with some works reported literature, where authors reported that cell viability was affected by BGP solution in a dose-dependent manner, due to a rapid release of BGP when immersed in medium, leading to hypertonicity of the medium ${ }^{35}$.

Last decade studies indicated that paracrine factors released by articular chondrocytes and their surface receptors were able to induce the chondrogenesis of MSCs, when in close-contact co-culture and deposit a cartilage specific matrix ${ }^{23}$. Therefore, in this work we designed a close-contact co-culture of hACHs spheroid on a MSCs-laden hydrogel for avoiding the chondrocytes issues of de-differentiation in fibroblasts when $2 \mathrm{D}$ cultured ${ }^{36}$. As shown in Fig. 5 the chondrocyte spheroid in co-culture maintained its shape at day 7 and day 14 in co-culture conditions while the chondrocyte spheroid in mono-culture disaggregated before 14 days, with the lack of stimulus from the MSCs considered to have led to the disaggregation. Furthermore, in the co-culture, many cells were observed in the proximity of the spheroid, rather than in other hydrogel areas and at 14 days all the nuclei appeared agglomerated in the spheroid, suggesting communication and migration of MSCs to the hACHs spheroid $^{37}$. Moreover, immunofluorescence results confirmed that the interaction between chondrocytes and MSCs in the co-culture allowed a wide presence of CD44 marker after 7 and 14 days, both into and around the spheroid and Col II (increasing from day 1 to day 28 as shown in Fig. S5), compared to the monoculture ${ }^{38,39}$. The benefit arising from close-contact co-culture was shown by histological analysis at 28 days, showing GAG accumulation and enhanced collagen production, uniformly distributed throughout the co-culture, with no evidence of this in the hACH mono-cultures (Fig. 6) or the MSC mono-cultures (Fig. S6b) ${ }^{40}$. After 45 days of culture round chondrocytes were uniformly distributed into the $\mathrm{CH} / \mathrm{BGP}$ hydrogel in both the co-culture and mono-culture, residing in the cavities or by the edges of the cavities (Fig. 6). A thin fiber-like matrix can be observed, secreted by the cells with an effective interaction visible between cells and matrix ${ }^{41}$. Overall the model has shown that chondrocytes are positively influenced by MSCs in a co-culture, even when the MSCs are not in direct contact, and with initial signalling between cell types inhibited through gel encapsulation of the MSCs.

\section{Experimental Section}

Materials. Unless otherwise stated, reagents were obtained from Sigma-Aldrich, UK. The ultrapure water employed $\left(\mathrm{dH}_{2} \mathrm{O}\right)$ throughout the experiments was obtained with a Milli-Q Integral system equipped with a BioPak ultrafiltration cartridge (Millipore, Merck).

Hydrogels preparation. A sterile $3.6 \% \mathrm{w} / \mathrm{v}$ chitosan solution ( $\mathrm{Mw}=100 \mathrm{kDa}$, DD $95 \%$; HMC + , Germany) was prepared adding slowly $\mathrm{CH}$ to $0.2 \mathrm{M}$ hydrochloric acid ( $\mathrm{HCl}$; reagent grade $37 \%$ ) solution, autoclaved at $121^{\circ} \mathrm{C}$ for $20 \mathrm{~min}$, left under stirring until achieving achieve an homogenous solution at room temperature (RT) and, then stored at $5^{\circ} \mathrm{C}$. For the preparation of the thermo-sensitive hydrogel, $1104.5 \mathrm{mg}$ of $\beta$-glycerophosphate disodium salt pentahydrate (Santa Cruz Biotec, US) were dissolved in $1 \mathrm{ml}$ of sterile DPBS using a vortex (IKA, 
Germany) for few minutes; Dulbecco's Modified Eagle's Medium (DMEM) supplemented with 10\% fetal bovine serum (FBS) and $5000 \mathrm{U} / \mathrm{mL}$ penicillin/streptomycin (P/S), was added to the salt solution in order to reach a final volume of $2.2 \mathrm{ml}$ and then kept at $5^{\circ} \mathrm{C}$ for 15 minutes. Finally, the prepared BGP solution was added drop-by-drop to the $\mathrm{CH}$ solution $(5 \mathrm{ml})$ in an ice bath, under stirring to obtain a final concentration of $2.5 \% \mathrm{w} / \mathrm{v}$. The $\mathrm{CH} / \mathrm{BGP}$ solution was poured in 48 -well plate and let it becoming gel at $37^{\circ} \mathrm{C}$ in incubator (SANYO MCO-18M Multigas Incubator). Then gels were cut in disks (3-4 $\mathrm{mm}$ height $\mathrm{x} 6 \mathrm{~mm}$ diameter) for further characterisation and stored at $5{ }^{\circ} \mathrm{C}$. A scheme of the hydrogel preparation and gelation process has been reported in Supplementary Data (Fig. S1).

Gelation time and thermal irreversibility. Hydrogel gelation time was measured at three different temperatures $\left(25,37,50^{\circ} \mathrm{C}\right)$ using the test tube inverting method ${ }^{19} . \mathrm{CH} / \mathrm{BGP}$ solutions $(1 \mathrm{ml}$ per bijou vial) were placed in a water bath to measure the gelation time at 37 and $50^{\circ} \mathrm{C}$ or maintained at room temperature (RT) to measure it at $25^{\circ} \mathrm{C}$. The samples flowability was observed every $30 \mathrm{~s}$ by tilting the vials, considering the gelation time when the flow of the solution stopped. The same test was utilised to study the thermal reversibility of the gelation according the protocol proposed by Ganji et al. ${ }^{22}$. The obtained $\mathrm{CH}$-based hydrogels were then cooled at a temperature of $4{ }^{\circ} \mathrm{C}$ or $25^{\circ} \mathrm{C}$. If the gels returned to a liquid solution, they were defined thermo-reversible hydrogels; otherwise the systems were considered thermo-irreversible.

Water uptake analysis. For the water uptake (WU) analysis, hydrogels were frozen at $-20^{\circ} \mathrm{C}$ overnight and then lyophilised for $48 \mathrm{~h}$ in a freeze-dryer (Alpha 1-2 LDplus, CHRIST, Germany), weighted and placed separately in a $5 \mathrm{ml}$ bijou vial containing $5 \mathrm{ml}$ of DPBS and stored at $37^{\circ} \mathrm{C}$. The weight of all the samples was measured before immersion and after $30 \mathrm{~min}, 1,2,4,6,24$ and $48 \mathrm{~h}$ of incubation. At each time, the samples were weighted after gently drying the extra DPBS on the surface using tissue papers. The water uptake percentage was calculated using the following equation:

$$
W U(\%)=\frac{W_{t}-W_{i}}{W_{i}} \times 100
$$

where $\mathrm{W}_{\mathrm{i}}$ is the initial weight of the hydrogel and $\mathrm{W}_{\mathrm{t}}$ is the weight of the hydrogel after the specific time point.

Nutrients uptake and release. A glucose solution was prepared with the light sensitive 2-NBDG, dissolved in DPBS $(0.06845 \mathrm{mg} / \mathrm{ml})$ according the protocol proposed by Ribeiro-Conceicao et al. ${ }^{42}$. To test the glucose uptake a qualitative analysis was performed: $2 \mathrm{ml}$ of $\mathrm{CH} / \mathrm{BGP}$ solution was let to gelate in a $5 \mathrm{ml}$ vial and then, $500 \mu \mathrm{l}$ of 2 -NBDG solution was added to the vial and observed over time. The time to obtain a complete yellow colour gel corresponded to the nutrients absorption time. For assessing the release over time, freeze dried samples were weighted, washed with DPBS and placed in a 48 -well plate where $2 \mathrm{ml} 2$-NBDG solution was added to each gel during different time-points ( $30 \mathrm{~min}, 1,3,6,24$ and $48 \mathrm{~h}$ ). At each time-point, gels were transferred to a new well-plate with DPBS to release the absorbed 2-NBDG. The released glucose solution was read at an excitation/emission of 465/540 nm using a LS-50B Luminescence Spectrometer (Perkin Elmer, Waltham, US).

Morphological analysis. Freeze-dried hydrogels were investigated before and after water uptake analysis by E-SEM (XL30 FEG Philips) at accelerating voltage of $10 \mathrm{kV}$. The samples were cut into small squares ( $2 \mathrm{~mm}$ diameter $\mathrm{x} 1 \mathrm{~mm}$ height), fixed on the aluminium stub using carbon tape and gold-coated using a BIO-RAD Sputter Coater machine. For pore size evaluation E-SEM images were analysed using an image software (ImageJ). Three images for sample type were analysed measuring 50 pores for each one. The pore size was averaged to give a mean pore size assuming all pores were circular.

Chemical characterisation. For the infrared spectroscopy $\mathrm{CH}$ and BGP raw powders and CH/BGP freeze-dried hydrogels (before and after water uptake) were analysed with a Spectrum Two PE instrument equipped with a horizontal attenuated total reflectance (ATR) crystal (ZnSe) (PerkinElmer Inc., US). Each spectrum was collected in absorbance mode as result of the average of 16 scans with $4 \mathrm{~cm}^{-1}$ resolution. Measurements were recorded in the wavelength range of $4000-550 \mathrm{~cm}^{-1}$.

Furthermore freeze dried hydrogels were examined by a scanning microprobe Kratos Axis Ultra-DLD XPS spectrometer (EPSRC service Cardiff, UK), equipped with a monochromatised AlK $\alpha$ X-ray radiation source. For each specimen, survey scans (Fixed Analyser Transmission mode, binding energy (BE) range 0-1200 eV, pass energy $117.4 \mathrm{eV})$ and high-resolution spectra were acquired of carbon $\left(\mathrm{C}_{1 \mathrm{~s}}\right)$, nitrogen $\left(\mathrm{N}_{1 \mathrm{~s}}\right)$, sodium $\left(\mathrm{Na}_{1 \mathrm{~s}}\right)$, oxygen $\left(\mathrm{O}_{1 \mathrm{~s}}\right)$, phosphorous $\left(\mathrm{P}_{2 \mathrm{p}}\right)$. Atomic concentration (At.\%) on the survey scan was performed using the built-in CasaXPS software package and in order to detect the BE representing the chemical binding states of the each elements within the films, the XPS spectra for the chemical elements detected from the films were subjected to peak deconvolution using the same software.

Compression and stress-relaxation tests. Compression tests were performed using a universal testing machine (EZ-SX, Shimadzu, Japan) equipped with a $20 \mathrm{~N}$ load cell and crosshead speed set at $1 \mathrm{~mm} \cdot \mathrm{min}^{-1}$. The compressive Young's moduli (E) were calculated as the slope of the initial elastic region of the curve $(0-10 \%$ strain). Then, stress-relaxation properties were evaluated following the protocol proposed by Bian et al., using a single compression ramp at a speed of $10 \% \mathrm{~min}^{-1}$ until reaching $10 \%$ strain $^{33}$. Subsequently, the strain was held constant for $1000 \mathrm{~s}$, while the load was recorded as a function of time. The equilibrium Young's modulus $\left(\mathrm{E}_{\mathrm{Y}}\right)$ was determined by the equilibrium load obtained after $1000 \mathrm{~s}$ of relaxation under unconfined strain. The data obtained were analysed using MATLAB R2017 software ${ }^{43}$. By fitting a third order exponential decay to the relaxation curves, obeying the generalized Maxwell model, three relaxation times were acquired, as: 


$$
\sigma(t)=A(e)^{-t / \tau_{1}}+B(e)^{-t / \tau_{2}}+C(e)^{-t / \tau_{3}}+D
$$

where A1, A2 and A3 were the amplitudes corresponding to the three different relaxation times $\tau 1, \tau 2$ and $\tau 3$.

Rheological analysis. Rheological measurements were performed on an oscillatory and rotational Anton Paar's Modular Compact Rheometer (MCR 302) using circular (diameter $5 \mathrm{~cm}$ ) disks. The CH/BGP solution was prepared at $4{ }^{\circ} \mathrm{C}$ and poured between the two plates for the analysis. The Strain Sweep test was done to verify the values of the strain amplitude in order to ensure that all the measurements were performed within the linear viscoelastic region (were $\mathrm{G}^{\prime}$ and $\mathrm{G}^{\prime \prime}$ were independent of the strain amplitude being linear and parallel between each other's) at $37^{\circ} \mathrm{C}$ with a rotational oscillation frequency of $1 \mathrm{~Hz}$. The Temperature Sweep test was performed to determine the exact $\mathrm{CH} / \mathrm{BGP}$ sol/gel transition temperature and this value is determined by $\mathrm{G}^{\prime}$ and $\mathrm{G}^{\prime \prime}$ curves crossing. The oscillatory measurement was set at a frequency of $1 \mathrm{~Hz}, 1 \%$ strain amplitude (linear region of the strain sweep tests) and with a temperature rate of $5^{\circ} \mathrm{C} / \mathrm{min}$ in the range of $0-50^{\circ} \mathrm{C}$. The Time Sweep Test was done to know the exact sol/gel transition time and this value is known when $\mathrm{G}^{\prime}$ and $\mathrm{G}^{\prime \prime}$ cross. To determine the gelation time, oscillatory measurements at $1 \mathrm{~Hz}$ and $1 \%$ strain, were started just after introducing cold solutions (at $4^{\circ} \mathrm{C}$ ) into the rheometer chamber pre-heated at $37^{\circ} \mathrm{C}$. The temperature was constant during the test at the same value.

Cells culture and encapsulation protocol. Human TERT immortalised bone marrow stromal cell line was kindly supplied by Prof P. Genever (York University) at passage 84 and cultured as reported ${ }^{44}$. Briefly, cells were grown at $37^{\circ} \mathrm{C}, 5 \% \mathrm{CO}_{2}$, in DMEM with low glucose content, supplemented with $10 \% \mathrm{FBS}, 2 \mathrm{mM}$ L-glutamine and a $1 \% \mathrm{P} / \mathrm{S}$. After the expansion, cells were used between passage 86 and 90. CH/BGP solution $(200 \mu \mathrm{L})$ was poured into a membrane-based cell culture insert (membrane pore size of $8.0 \mu \mathrm{m}$, Merck, Millipore, Germany) at room temperature and Y201 MSCs were added and mixed gently with the CH/BGP solution at an optimised cellular density of $2 \times 10^{6} \mathrm{cell} \mathrm{ml}^{-1}$ following the consideration reported also by Liu et al. ${ }^{45}$. Samples were placed in 24 -well plates and allowed to gelate in the incubator $\left(37^{\circ} \mathrm{C}\right.$ and $\left.5 \% \mathrm{CO}_{2}\right)$. Finally, after $30 \mathrm{~min}$, $1 \mathrm{ml}$ of fresh DMEM was added into each well and refreshed three times per week. Human articular chondrocytes (hACHs) were cultured as recommended by the seller. Cells were cultured in Chondrocyte Growth Medium ready-to-use (PromoCell, UK) at $37^{\circ} \mathrm{C}$ in a humidified atmosphere incubator containing $5 \% \mathrm{CO}_{2}$ and sub-cultured to passage 5-6 for the experiments.

Co-culture assessment. To set up a direct-contact co-culture system, a chondrocyte spheroid was seeded on the MSCs-laden hydrogel, supplemented with chondrocytes growth media and compared with a mono-culture made of a chondrocytes spheroid seeded on plain hydrogel (without MSCs). For spheroids formation, hACHs dispersed in chondrocytes growth medium supplemented with $0.25 \%$ Methylcellulose were seeded in a round bottom 96 -wells plate (non-tissue culture treated) at a density of $2 \times 10^{5}$ cells/well ${ }^{44,46}$. After one day, the hydrogels were prepared following the same protocol, but using $100 \mu \mathrm{L}$ of solution with the same MSCs cell density $\left(2 \times 10^{6} \mathrm{cell} \mathrm{ml}^{-1}\right)$. After formation of the hydrogels, spheroids were aspired from the 96-well plate and placed on the top of the respective gels and the respective media were added. Further controls of MSCs mono-cultures, supplemented with chondrogenic media or chondrocytes growth media were analysed (reported in the Supporting Information).

Cytotoxicity assay. TEM (Philips CM 100 Compustage FEI) was used for investigating the polymer localization and cell structure (organelles and internal structure) at voltage of $100 \mathrm{kV}$. Digital images were collected using an AMT CCD camera (Deben). Prior to the analysis, cells encapsulated in the hydrogels were fixed overnight using a pre-warmed solution of $2 \%$ glutaraldehyde (TAAB Laboratory Equipment) in sodium cacodylate buffer at $4{ }^{\circ} \mathrm{C}$, followed by a post-fixation with $1 \%$ osmium tetroxide (Agar Scientific). After various dehydration steps, hydrogels were embedded in resin, and cut in ultrathin sections using a diamond knife on a Leica EM UC7 ultra microtome (Leica Microsystems). The sections were stretched with chloroform to eliminate compression, mounted on Pioloform-filmed copper grids (Agar Scientific) and ready to be visualised. Further cytotoxicity assessment are reported in Supporting Information.

Hydrogel cytocompatibility. Live/Dead assay (LIVE/DEAD ${ }^{\circledR}$ Cell Imaging Kit, Life Technologies, UK) was used according to the manufacturer's instructions. This fluorescence-based kit combines calcein AM and ethidium bromide to yield two-colour discrimination of the population of live cells (green) from the dead cells (red). Each cell culture condition was washed twice with PBS before incubation with staining. In brief, $4 \mu \mathrm{M}$ ethidium homodimer- 1 and $10 \mu \mathrm{M}$ calcein dilute in DPBS, were incubated with the cell-encapsulated samples for $30 \mathrm{~min}$ at $37^{\circ} \mathrm{C}^{47}$. Images were collected at 1 and 3 days using a Nikon A1R inverted confocal microscope ${ }^{48}$.

To test the cells metabolic activity, culture medium was removed at each time point (1,3 and 7 days), samples were washed with DPBS and $1 \mathrm{ml}$ of PrestoBlue ${ }^{\mathrm{TM}}$ reagent (Thermo Scientific, UK) diluted in DMEM (1:10) protected from light; was added to each well with the gel and incubated for $2.5 \mathrm{~h}$ at $37^{\circ} \mathrm{C}$ and $5 \% \mathrm{CO}_{2}$. Then, $200 \mu \mathrm{l}$ of each well solution (in triplicate) was transferred to a white bottom 96-well plate and a LS-50B Luminescence Spectrometer (Perkin Elmer, Waltham, MA) was used to measure the fluorescence (excitation/emission of $560 / 590 \mathrm{~nm}$ ). Then, samples were washed with PBS twice and fresh media was added for the next time point.

For immunostaining analysis, samples were fixed in pre-warmed $4 \% \mathrm{w} / \mathrm{v}$ paraformaldehyde (PFA) and cells were consequently permeabilised using $0.1 \% \mathrm{v} / \mathrm{v}$ Tween $20^{\circledR}$ in DPBS for three washes. Rhodamine-phalloidin was prepared using phalloidin-tetramethylrhodamine B isothiocyanate $\left(1: 1000\right.$ in $0.1 \%$ DPBS/Tween $\left.20^{\circledR}\right)$ for $20 \mathrm{~min}$ at RT. Then, samples were washed with $0.1 \% \mathrm{DPBS} /$ Tween $20^{\circledR}$ solution and immersed in DAPI solution (Vector Laboratories,UK) (1:2500 in 0.1\% DPBS/Tween20 $\left.{ }^{\circledR}\right)$ for $10 \mathrm{~min}$ at RT. Experiments were light sensitive and images were collected at 1 and 3 days using a Nikon A1R inverted confocal microscope. 
Co-culture immunofluorescence analysis. Co-culture samples were fixed as for the immunostaining, blocked for $1 \mathrm{~h}$ in DPBS supplemented with $2 \% \mathrm{BSA}$ at $4{ }^{\circ} \mathrm{C}$ and incubated overnight with primary antibodies: polyclonal Anti-Collagen II (Anti-Col II; ab34712, abcam) diluted in 0.1\% DPBS/Tween20 ${ }^{\circledR}$ (1:200) and monoclonal Anti-CD44 (ab189524, abcam) diluted in 0.1\% DPBS/Tween20 ${ }^{\circledR}$ (1:250), following the product datasheets $^{49}$. Samples were washed twice with $0.1 \%$ DPBS/Tween $20^{\circledR}$ for 5 min and incubated with Anti-Col II secondary antibody, Alexa Fluor ${ }^{\circledR}$ goat anti-rabbit igG $(\mathrm{H}+\mathrm{L})$ (ab150080, abcam) diluted in $0.1 \%$ DPBS/ Tween $20^{\circledR}(1: 1000)$ for $1 \mathrm{~h}$ at RT. Then, samples were washed twice with $0.1 \%$ DPBS/Tween $20^{\circledR}$ for 5 min and incubated with Anti-CD44 secondary fluorescein-labelled goat anti-rabbit IgG (H+L) (F2765, Thermo Fisher Scientific) diluted in $0.1 \%$ DPBS/Tween $20^{\circledR}(1: 1000)$, for $1 \mathrm{~h}$ at RT. Sample were re-washed twice with $0.1 \%$ DPBS/ Tween $20^{\circledR}$ and DAPI staining for nuclei was performed (as explained before) and imaged using a Nikon A1R inverted confocal microscope at 7 and 14 days.

Co-culture histological analysis. Co-culture samples were fixed in pre-warmed 10\% formalin (24h), and transferred to $70 \%$ Ethanol (EtOH) (Thermo Fisher Scientific). Then, samples were transferred to histology cassettes lined with biopsy sponges and dehydrate in graded ethanol series (80\%, 95\% and 100\% EtOH) for $30 \mathrm{~min}$ each, followed by two clarification steps in xylene (Thermo Fisher Scientific) ${ }^{50}$. Following this, samples were paraffin-embedded following routine histological procedures and sectioned in three $5 \mu \mathrm{m}$-thick slices. The slices were fixed on glass slides, deparaffined and then stained for the collagen and GAGs production analysis. In order to evaluate GAGs production, Alcian Blue acid mucins staining was done. Slides were washed in running water and stained for $20 \mathrm{~min}$ with Alcian Blue solution ( $\mathrm{pH}$ 5), prepared by dissolving $5 \mathrm{mg}$ of Alcian Blue $8 \mathrm{GX}$ (Merck) in $500 \mathrm{ml} \mathrm{dH}_{2} \mathrm{O}$. Then, slides were rinsed in running $\mathrm{dH}_{2} \mathrm{O}$. The amount of collagen deposited by the cells was investigated using the Sirius Red assay ${ }^{51}$. Sections, were washed in two changes of acidified water, made diluting $0.5 \mathrm{ml}$ of glacial acetic acid in $100 \mathrm{ml}$ of $\mathrm{dH}_{2} \mathrm{O}$. Then, samples were treated with Sirius-red/picric acid solution (Sircol ${ }^{\mathrm{TM}}$, Biocolor Ltd., UK) for $1 \mathrm{~h}$ at room temperature and re-washed in two changes of acidified water. Slides underwent a dehydration process in three rapid changes of absolute alcohol and cleared in Xylene and mounted in DPX Mounting for histology. Stained slides were covered with a glass slide and imaged with Leica Stereomicroscope (Bright field) and analysed with Leica software.

Co-culture morphological analysis. The morphology of the cells within the scaffolds were observed using SEM after 28days of co-culture. Samples were fixed in pre-warmed 2\% Glutaraldehyde overnight, rinsed in PBS twice and dehydrated in ethanol grades: $30 \mathrm{~min}$ in each $25 \%, 50 \%$ and $75 \% \mathrm{EtOH}$ and $1 \mathrm{~h}$ in $100 \% \mathrm{EtOH}$ (twice). Samples were stored at $4^{\circ} \mathrm{C}$ in $100 \% \mathrm{EtOH}$ until critical point dried using a BAL-TEC 030 Critical Point Dryer (Leica Geosystems Ltd, Milton Keynes, UK). Finally, gels were mounted on carbon discs (TAAB Laboratory Equipment) and gold-coated using a Polaron E5000 SEM Coating unit (Quorum Technologies Ltd, UK). After gold coating using Polaron SEM coating unit with $15 \mathrm{~nm}$ of gold samples were imaged at different magnifications. SEM images for MSCs controls are reported in the Supporting Informations (Fig. S6).

Statistical analysis. Tests were performed at least in triplicate for each sample. The results were represented as mean \pm standard deviation. Differences between groups were determined using One-way analysis of variance (ANOVA) with Turkey's multiple comparison test using levels of statistical significance of $\mathrm{p}<0.05(*)$.

\section{Conclusion}

The fabricated thermo-sensitive chitosan-based hydrogels presented fast sol/gel transition time that allows cell encapsulation, an intrinsic porous structure, and a high fluid uptake capability. The gel based co-culture system allows for the influence of two cell types on one another to be investigated with the gel offering tissue-like diffusion rates of cell signalling molecules, and showed that encapsulated MSCs stimulate chondrocyte activity within a gel co-culture, both in terms of maintaining the coherence of chondrocyte spheroids and leading to enhanced collagen production. A further investigation could be the assessment of the hydrogel system as in vitro model for mimicking native cartilage tissue for e.g. studying pathological processes or drug screening or as in vivo carrier in order to transplant the chondrocytes spheroid to the target site immediately after formation via surface entrapment onto the $\mathrm{CH} / \mathrm{BGP}$ hydrogel.

\section{Data Availability}

The datasets generated and/or analysed during the current study are available from the corresponding author on reasonable request. See Supplementary Information for cytotoxicity tests on BGP, representative stress-strain curves and rheology plots on CH/BGP hydrogels, and biological assessment of the monoculture of Y201 MSCs.

\section{References}

1. Sophia Fox, A. J., Bedi, A. \& Rodeo, S. A. The basic science of articular cartilage: Structure, composition, and function. Sports Health 1, 461-468 (2009).

2. Taylor, D. W. et al. Hyaline Cartilage Tissue Is Formed through the Co-culture of Passaged Human Chondrocytes and Primary Bovine Chondrocytes. J. Histochem. Cytochem. 60, 576-587 (2012).

3. Filardo, G., Perdisa, F., Roffi, A., Marcacci, M. \& Kon, E. Stem cells in articular cartilage regeneration. J. Orthop. Surg. Res. 11 (2016).

4. Oseni, A. O., Butler, P. E. \& Seifalian, A. M. Optimization of chondrocyte isolation and characterization for large-scale cartilage tissue engineering. J. Surg. Res. 181, 41-48 (2013).

5. Benya, P. D. \& Shaffer, J. D. Dedifferentiated chondrocytes reexpress the differentiated collagen phenotype when cultured in agarose gels. Cell 30, 215-224 (1982).

6. Ullah, I., Subbarao, R. B. \& Rho, G. J. Human mesenchymal stem cells - current trends and future prospective. Biosci. Rep. 35, 1-18 (2015).

7. Zhang, Y. et al. Co-culture systems-based strategies for articular cartilage tissue engineering. J. Cell. Physiol. 233, 1940-1951 (2018).

8. Wu, L. et al. Trophic Effects of Mesenchymal Stem Cells Increase Chondrocyte Proliferation and Matrix Formation. Tissue Eng. Part A 17, 1425-1436 (2011). 
9. Bogdanowicz, D. R. \& Lu, H. H. Multifunction Co-culture Model for Evaluating Cell-Cell Interactions. Methods Mol Biol. 1202, 29-36 (2014)

10. Heng, B. C., Cao, T. \& Lee, E. H. Directing Stem Cell Differentiation into the Chondrogenic Lineage In Vitro. Stem Cells 22, 1152-1167 (2004).

11. Ahmed, N., Dreier, R., Göpferich, A., Grifka, J. \& Grässel, S. Soluble signalling factors derived from differentiated cartilage tissue affect chondrogenic differentiation of rat adult marrow stromal cells. Cell. Physiol. Biochem. 20, 665-678 (2007).

12. Amer, M. H., Rose, F. R. A. J., Shakesheff, K. M. \& White, L. J. A biomaterials approach to influence stem cell fate in injectable cellbased therapies. 1-15 (2018).

13. Caló, E. \& Khutoryanskiy, V. V. Biomedical applications of hydrogels: A review of patents and commercial products. Eur. Polym. J. 65, 252-267 (2015).

14. Chenite, A. et al. Novel injectable neutral solutions of chitosan form biodegradable gels in situ. Biomaterials 21, 2155-2161 (2000).

15. Zhou, H. Y., Jiang, L. J., Cao, P. P., Li, J. B. \& Chen, X. G. Glycerophosphate-based chitosan thermosensitive hydrogels and their biomedical applications. Carbohydr. Polym. 117, 524-536 (2015).

16. Tahrir, G. F., Ganji, F. \& Ahooyi, T. Injectable Thermosensitive Chitosan/Glycerophosphate-Based Hydrogels for Tissue Engineering and Drug Delivery Applications: A Review. Recent Pat. Drug Deliv. Formul. 9, 107-120 (2015).

17. Duarte Campos, D. F., Drescher, W., Rath, B., Tingart, M. \& Fischer, H. Supporting Biomaterials for Articular Cartilage Repair. Cartilage 3, 205-221 (2012).

18. Skwarczynska, A. et al. The structural (FTIR, XRD, and XPS) and biological studies of thermosensitive chitosan chloride gels with $\beta$-glycerophosphate disodium. J. Appl. Polym. Sci. 135, 1-8 (2018).

19. Nafee, N., Zewail, M. \& Boraie, N. Alendronate-loaded, biodegradable smart hydrogel: a promising injectable depot formulation for osteoporosis. J. Drug Target. 0, 1-13 (2017).

20. Salis, A. et al. Development of thermosensitive chitosan/glicerophospate injectable in situ gelling solutions for potential application in intraoperative fluorescence imaging and local therapy of hepatocellular carcinoma: a preliminary study. Expert Opin. Drug Deliv. 12, 1583-1596 (2015)

21. Szymańska, E. et al. The effect of $\beta$-glycerophosphate crosslinking on chitosan cytotoxicity and properties of hydrogels for vaginal application. Polymers (Basel). 7, 2223-2244 (2015).

22. Ganji, F., Abdekhodaie, M. J. \& Ramazani, A. Gelation time and degradation rate of chitosan-based injectable hydrogel. J. Sol-Gel Sci. Technol. 42, 47-53 (2007).

23. Supper, S. et al. Thermosensitive chitosan/glycerophosphate-based hydrogel and its derivatives in pharmaceutical and biomedical applications. Expert Opin. Drug Deliv. 11, 249-267 (2014).

24. Skwarczynska, A. L., Kuberski, S., Maniukiewicz, W. \& Modrzejewska, Z. Thermosensitive chitosan gels containing calcium glycerophosphate. Spectrochim. Acta - Part A Mol. Biomol. Spectrosc. 201, 24-33 (2018).

25. Loh, Q. L. \& Choong, C. Three-Dimensional Scaffolds for Tissue Engineering Applications: Role of Porosity and Pore Size. Tissue Eng. Part B Rev. 19, 485-502 (2013).

26. M Iliescu, C. D., Hoemann, M. S., Shive, A. \& Chenite, M. D. B. Ultrastructure of Hybrid Chitosan-Glycerol Phosphate Blood Clots by Environmental Scanning Electron Microscopy. Microsc. Res. Tech. 71, 236-247 (2008).

27. Sci, P. P. Advancing biomaterials of human origin for tissue engineering. 86-168, https://doi.org/10.1016/j.progpolymsci.2015.02.004. Advancing (2017).

28. Kelly, T. A. N. et al. Tissue-engineered articular cartilage exhibits tension-compression nonlinearity reminiscent of the native cartilage. J. Biomech. 46, 1784-1791 (2013).

29. Kocen, R., Gasik, M., Gantar, A. \& Novak, S. Viscoelastic behaviour of hydrogel-based composites for tissue engineering under mechanical load. Biomed. Mater. 12 (2017).

30. Visser, J. et al. Reinforcement of hydrogels using three-dimensionally printed microfibres. Nat. Commun. 6, 1-10 (2015).

31. Wagenseil, J. E., Wakatsuki, T., Okamoto, R. J., Zahalak, G. I. \& Elson, E. L. One-Dimensional Viscoelastic Behavior of Fibroblast Populated Collagen Matrices. J. Biomech. Eng. 125, 719 (2003).

32. Nguyen, Q. T. \& Sah, R. L. Cartilage-like mechanical properties of poly (ethylene glycol)- diacrylate hydrogels. Biomaterials 33, 6682-6690 (2012).

33. Bian, L., Zhai, D. Y., Mauck, R. L. \& Burdick, J. A. Coculture of Human Mesenchymal Stem Cells and Articular Chondrocytes Reduces Hypertrophy and Enhances Functional Properties of Engineered Cartilage. Tissue Eng. Part A 17, 1137-1145 (2011).

34. Kumari, S., Mg, S. \& Mayor, S. Endocytosis unplugged: Multiple ways to enter the cell. Cell Res. 20, 256-275 (2010).

35. Ahmadi, R. \& De Bruijn, J. D. Biocompatibility and gelation of chitosan-glycerol phosphate hydrogels. J. Biomed. Mater. Res. - Part A 86, 824-832 (2008).

36. Nazempour, A. \& Van Wie, B. J. Chondrocytes, Mesenchymal Stem Cells, and Their Combination in Articular Cartilage Regenerative Medicine. Ann. Biomed. Eng. 44, 1325-1354 (2016).

37. Somoza, R. A., Welter, J. F., Correa, D. \& Caplan, A. I. Chondrogenic Differentiation of Mesenchymal Stem Cells: Challenges and Unfulfilled Expectations. Tissue Eng. Part B Rev. 20, 596-608 (2014).

38. Wu, L. et al. Human developmental chondrogenesis as a basis for engineering chondrocytes from pluripotent stem cells. Stem Cell Reports 1, 575-589 (2013).

39. Campbell, D. \& Pei, M. Surface Markers for Chondrogenic Determination: A Highlight of Synovium-Derived Stem Cells. Cells 1, $1107-1120$ (2012).

40. Richardson, S. M., Hughes, N., Hunt, J. A., Freemont, A. J. \& Hoyland, J. A. Human mesenchymal stem cell differentiation to NP-like cells in chitosan-glycerophosphate hydrogels. Biomaterials 29, 85-93 (2008).

41. Hong, Y. et al. Covalently crosslinked chitosan hydrogel: Properties of in vitro degradation and chondrocyte encapsulation. Acta Biomater. 3, 23-31 (2007).

42. da Conceicao Ribeiro, R. et al. Reactive jet impingement bioprinting of high cell density gels for bone microtissue fabrication. Biofabrication (2018).

43. Bonifacio, M. A. et al. Antibacterial effectiveness meets improved mechanical properties: Manuka honey/gellan gum composite hydrogels for cartilage repair. Carbohydr. Polym. 198, 462-472 (2018).

44. James, S. et al. Multiparameter Analysis of Human Bone Marrow Stromal Cells Identifies Distinct Immunomodulatory and Differentiation-Competent Subtypes. Stem Cell Reports 4, 1004-1015 (2015).

45. Liu, T. et al. Encapsulation of mesenchymal stem cells in chitosan/ $\beta$-glycerophosphate hydrogel for seeding on a novel calcium phosphate cement scaffold. Med. Eng. Phys. 56, 9-15 (2018).

46. Zhao, Z., Zhou, X., Guan, J., Wu, M. \& Zhou, J. Co-implantation of bone marrow mesenchymal stem cells and chondrocytes increase the viability of chondrocytes in rat osteo-chondral defects. Oncol. Lett. 15, 7021-7027 (2018).

47. Montalbano, G. et al. Synthesis of bioinspired collagen/alginate/fibrin based hydrogels for soft tissue engineering. Mater. Sci. Eng. C 91, 236-246 (2018).

48. Ribeiro, R. D. C. et al. Temporary Single-Cell Coating for Bioprocessing with a Cationic Polymer. ACS Appl. Mater. Interfaces $\mathbf{9}$, 12967-12974 (2017).

49. Berg, V. et al. Human articular chondrocytes express ChemR23 and chemerin; ChemR23 promotes inflammatory signalling upon binding the ligand chemerin21-157. Arthritis Res. Ther. 12, R228 (2010). 
50. Vemuri, M. C., Chase, L. G. \& Rao, M. S. Mesenchymal stem cell assays and applications. Methods in molecular biology (Clifton, N.J.) 698 (2011).

51. Jimenez, W. et al. Measurement of fibrosis in needle liver biopsies: Evaluation of a colorimetric method. Hepatol. Off. J. Am. Assoc. Study Liver Dis. 5, 815-818 (1985).

\section{Acknowledgements}

Annachiara Scalzone acknowledges support from EPSRC for her Ph.D. studentship (EPSRC Grant EP/ R51309X/1). The authors thank Dr K White for TEM images (Electron Microscopy Research Services), L Boyd and X Xu for Ultramicrotome sectioning (Medical School), Dr I A Garcia for E-SEM Images (Herschel Building) and P Melo for SEM images (School of Engineering) for their support at Newcastle University.

\section{Author Contributions}

A.S., A.M.F. and P.G. conceived and designed the experiments. A.S., C.T. and P.G. performed the experiments; A.S., A.F.M, and P.G. analysed the data. G.C. and K.D. contributed material preparation; A.S., A.M.F. and C.T. wrote the paper and K.D., G.C. and P.G. revised the paper drafts.

\section{Additional Information}

Supplementary information accompanies this paper at https://doi.org/10.1038/s41598-019-51070-7.

Competing Interests: The authors declare no competing interests.

Publisher's note Springer Nature remains neutral with regard to jurisdictional claims in published maps and institutional affiliations.

(i) Open Access This article is licensed under a Creative Commons Attribution 4.0 International License, which permits use, sharing, adaptation, distribution and reproduction in any medium or format, as long as you give appropriate credit to the original author(s) and the source, provide a link to the Creative Commons license, and indicate if changes were made. The images or other third party material in this article are included in the article's Creative Commons license, unless indicated otherwise in a credit line to the material. If material is not included in the article's Creative Commons license and your intended use is not permitted by statutory regulation or exceeds the permitted use, you will need to obtain permission directly from the copyright holder. To view a copy of this license, visit http://creativecommons.org/licenses/by/4.0/.

(C) The Author(s) 2019 